\title{
Challenges for cosmic-ray experiments
}

\author{
Thomas Gaisser ${ }^{\mathrm{a}}$ \\ Bartol Research Institute and Department of Physics and Astronomy, University of Delaware, Newark, DE 19716, USA
}

\begin{abstract}
This paper is a commentary on presentations at ISVHECRI 2016 related to cosmic-rays, gamma-rays and neutrinos. Its goal is to highlight the unanswered questions raised during the conference about the sources of these cosmic particles and the relations among them.
\end{abstract}

\section{Introduction}

A current theme of astro-particle physics is "multimessenger astronomy," which emphasizes use of gammarays and neutrinos to address still unanswered questions on the origin of high-energy cosmic rays. With this theme in mind, I review the presentations on cosmicray spectra and composition, gamma-ray astronomy and neutrino astronomy presented at ISVHECRI 2016. The role of hadronic interactions is addressed in the review paper of Tanguy Pierog [1].

\section{Some cosmic-ray questions}

Figure 1 gives a global view of the cosmic-ray spectrum. The abundant elements of the primary cosmicray spectrum are measured accurately to energies higher than a $\mathrm{TeV}$ per nucleon with spectrometers in space [2-4]. Calorimetric measurements with balloonborne detectors $[5,6]$ extend direct measurements to higher energy but with somewhat less precision. This means that we have good coverage of the composition with direct measurements up to about $100 \mathrm{TeV}$ energy per nucleus. Indirect measurements with large detectors on the surface are needed for the higher energy cosmic rays. There are several questions of current interest associated with the various features in the energy spectrum:

- What is the composition in the knee region and how does it connect with direct measurements at lower energy?

- What is the cause of the hardening of the spectrum around $20 \mathrm{PeV}$ ?

- Where is the transition from Galactic to extra-galactic cosmic rays and how is it related to composition around the ankle?

- What is responsible for the apparent end of the spectrum around $100 \mathrm{EeV}$ ?

- Does the difference between the Auger and the TA spectrum in the cutoff region show that the cosmic-ray spectrum is different in different regions of the sky?

a e-mail: gaisser@bartol.udel.edu

\subsection{The knee region}

Most air shower measurements have a threshold around a $\mathrm{PeV}$, while direct measurements extend only to $\sim 100 \mathrm{TeV}$. An exception is the ARGO-YBJ RPC carpet detector at high altitude in Tibet, which has the potential to cover the gap between direct and indirect measurements [8]. TAIGA-HISCORE is also starting to measure the spectrum down to $\sim 300 \mathrm{TeV}$ [9]. ARGO-YBJ have reported measurements of both the all-particle spectrum and the spectrum of the light $(\mathrm{p}+\mathrm{He})$ component [10]. The measurements cover the energy range from $\sim 10 \mathrm{TeV}$ to the knee region. While the measurement of the all-particle spectrum agrees with several other EAS measurements through the knee region, the light component appears to steepen starting around $700 \mathrm{TeV}[8,10]$. In contrast, KASCADE $[11,12]$ shows the proton steepening above a $\mathrm{PeV}$. In his presentation, DiSciascio compares the ARGJOYBJ result with the Hörandel parameterization [13] of $\mathrm{p}+\mathrm{He}$, which also steepens at higher energy. The IceCube/IceTop composition analysis [15] starts around $3 \mathrm{PeV}$, too high to provide insight on this question.

Indirect measurements of the composition with EAS detectors are difficult, and the ARGO-YBJ result points to an ambiguity that needs to be resolved. A practical aspect is its implication for the atmospheric neutrino flux at high energy relevant for IceCube. Calculation of the flux of atmospheric neutrinos depends on the spectrum of nucleons as a function of energy per nucleon, which is dominated by protons and helium. If the proton and helium components steepen at $700 \mathrm{GV}$, then there should be a compensating increase in heavier nuclei to keep the allparticle spectrum constant. The sketch in Fig. 2 illustrates the effect, which would likely be a suppression of the flux of nucleons in a range around a PeV that arises if the all-particle spectrum is dominated by heavy nuclei in this region. This in turn would significantly reduce the flux of muons and muon-neutrinos around $100 \mathrm{TeV}$.

\subsection{From the knee to the ankle}

Figure 3 (left) compares measurements of the spectrum by KASCADE-Grande [12,16], TUNKA-133 [17,18] and IceTop $[15,19,20]$. The solid line shows a spectrum with a constant differential index of -3 . The data show clear structure between the knee and the ankle, with a

(C) The Authors, published by EDP Sciences. This is an Open Access article distributed under the terms of the Creative Commons Attribution License 4.0 (http://creativecommons.org/licenses/by/4.0/). 


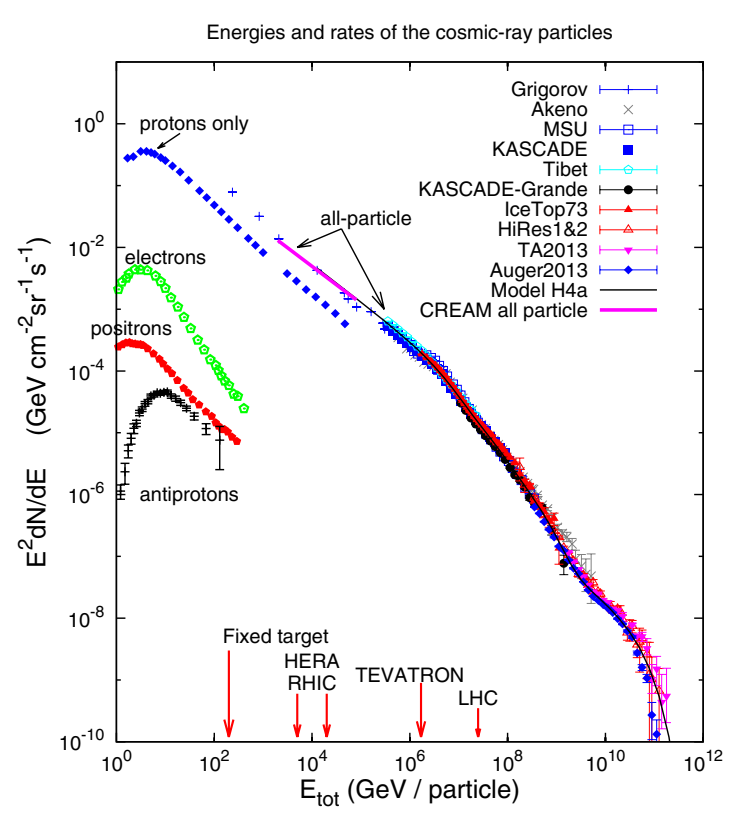

Figure 1. Overview of the energy spectra of various components of the cosmic radiation (Fig. 2.1 of [7]).

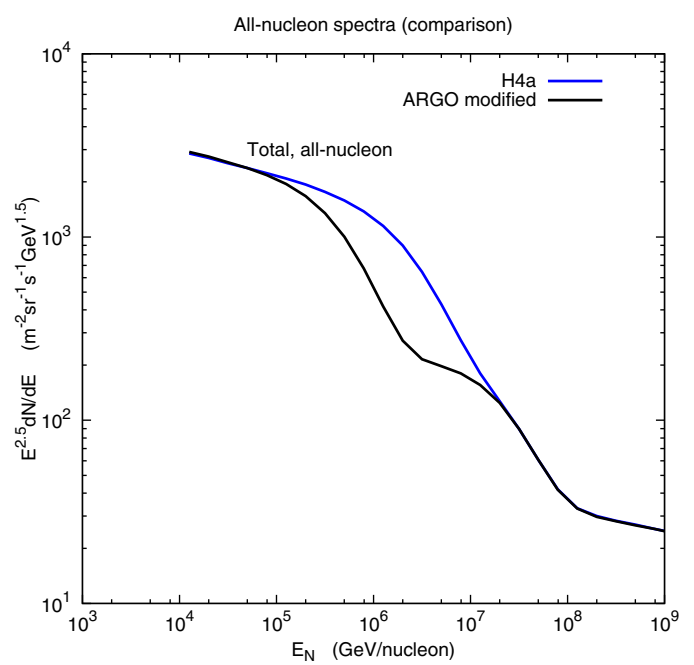

Figure 2. The spectrum of nucleons for the H4a model [14] compared with a modified version in which the cutoff rigidities for $\mathrm{p}$ and $\mathrm{HE}$ are reduced to $700 \mathrm{GV}$ and the all-particle spectrum is restored by increasing the contribution of the $\mathrm{CNO}$ and $\mathrm{Fe}$ groups.

hardening around $2 \times 10^{16} \mathrm{eV}$ and a second knee above $10^{17} \mathrm{eV}$. The KASCADE-Grande analysis uses the fraction of muons to separate the spectrum into light and heavy components [12,21]. The data suggest that the subdominant light component increases relative to the heavy component as energy increases toward the PeV region, as shown in Fig. 3 (right). A possible interpretation is that the increase of the light component reflects the population of cosmic rays from extragalactic sources, while the steeper heavy component is the end of the Galactic population.

\subsection{The highest energy cosmic rays}

Measurements of the cosmic-ray spectrum to the highest energy were presented at ISVHECRI 2016 from both Telescope Array (TA) [22] and Auger [23]. They are in excellent agreement with each other through the ankle region within their systematic uncertainties in energy. However, after shifting the Auger energy assignment up by $8 \%$ (or the TA spectrum down by a similar amount) the TA spectrum remains somewhat higher than the Auger spectrum above $10 \mathrm{EeV}$.

The question of composition of the highest energy cosmic rays has long been an important unresolved issue. Both TA and Auger find a large fraction of protons in the EeV range, above which the interpretations differed, with Auger preferring heavier and TA lighter composition. Inferences about composition are based on both the mean depth of shower maximum as a function of energy and on fluctuations in depth of maximum in each energy bin, and they depend on the hadronic interaction model used to make the interpretation. The TA presentation [22] includes a plot of mean depth of maximum for both experiments obtained by the joint composition working group that includes members of both experiments. The results of the two experiments are not inconsistent with each other. In comparison with the interaction model QGSJETII-03 the depth of maximum measurements are between protons and iron, but closer to the proton limit. Thus at present the composition at the highest energy remains an open question.

Composition from $1-100 \mathrm{EeV}$ is the key to what is one of the most important open questions in cosmicray physics, namely, the cause of the apparent cutoff in the spectrum at $100 \mathrm{EeV}$. There are two possibilities. If protons dominate at high energy, the natural interpretation would be the GZK process [24,25], energy loss to photo-pion production during propagation in the cosmic microwave background radiation. The other possibility is that the accelerators are reaching their maximum rigidity, as suggested by the Hillas plot [26]. The Auger presentation [23] illustrates the different energydependent compositions that characterize each of these possibilities [27]. The GZK explanation requires mostly protons at the highest energy while in the Hillas case an increasing fraction of heavy nuclei would be expected as the cutoff in rigidity affects protons first. In both cases effects of nuclear fragmentation during propagation must accounted for in addition to the source composition.

Both TA and Auger have initiated upgrades aimed at understanding the composition and the related question of anisotropy of the highest energy cosmic rays. TA $\times 4$ will expand the surface detector with two large scintillator arrays (grid spacing $2.08 \mathrm{~km}$ ) adjacent to the present array. One of the main aims is to get a better understanding of the hotspot observed in the TA data [28], which persists after seven years of observation.

The upgrade of the Pierre Auger Observatory [29] includes installation of a scintillator on top of each tank, improved electronics and underground muon detectors. With improved ability to separate the muon signal from the electromagnetic signal in the surface detector, the upgraded array surface array will have sensitivity to primary composition with $100 \%$ duty cycle. The goal is to determine the origin of the flux suppression at the highest energy and to determine the feasibility of astronomy with cosmic-ray protons.

\subsection{Cosmic-ray overview}

Using its low energy extension (TALE), the TA group has presented a combined spectrum that extends all the way 

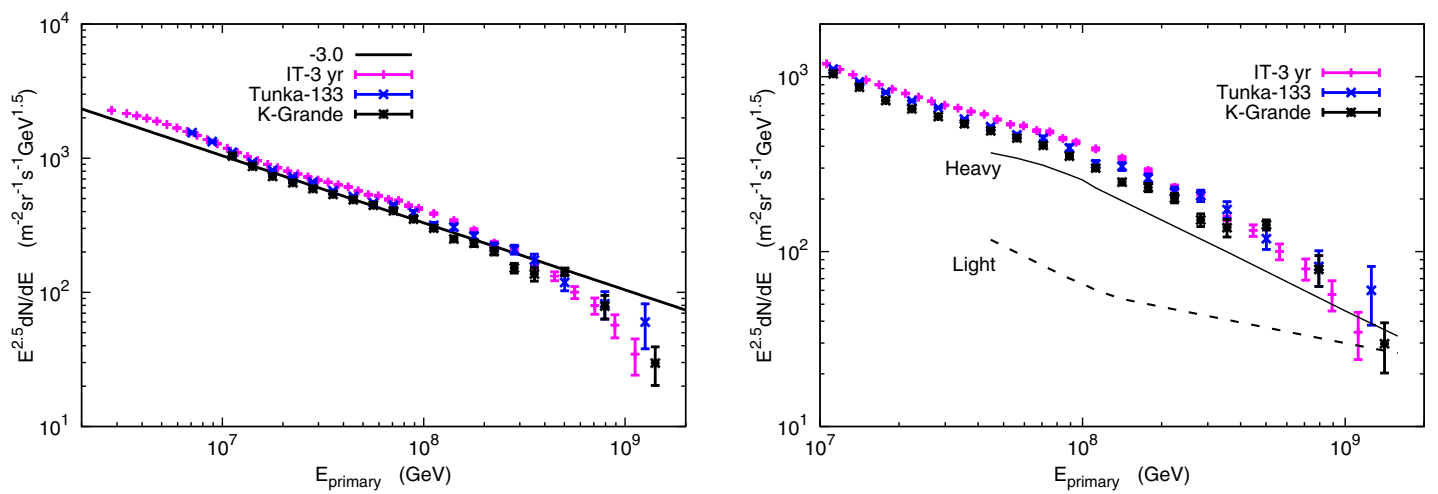

Figure 3. Measurements of the spectrum between the knee and the ankle, Left: compared to a constant $E^{-3}$ differential spectrum (Fig. 17.2 of [7]) and Right: showing the heavy and light fractions as measured by KASCADE-Grande [21] separately (Fig. 17.3 of [7]).

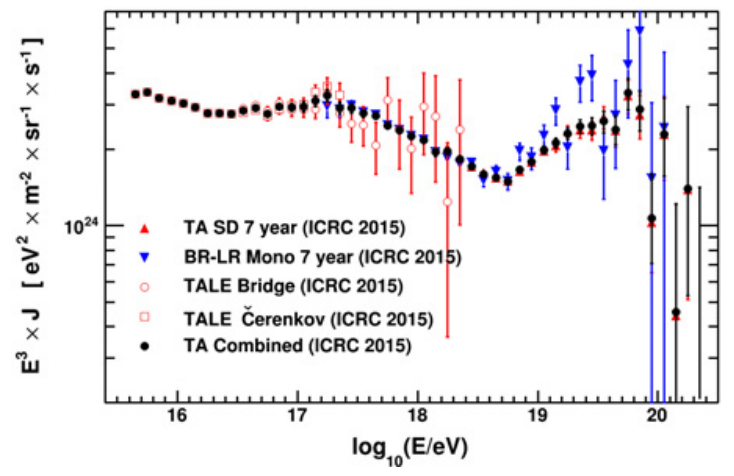

Figure 4. The combined spectrum of TA presented at this conference [22]) and shown here by kind permission of John Matthews.

down to $5 \mathrm{PeV}$ (Fig. 4). The data are consistent with those shown in Fig. 3 in the overlap region. The hardening of the spectrum at $2 \times 10^{16} \mathrm{eV}$ and the second knee just above $10^{17} \mathrm{eV}$ are clearly visible, as well as the ankle and the suppression above $10^{20} \mathrm{eV}$.

The composition also shows an energy dependence with features that may be correlated with the spectrum, as illustrated in Fig. 5 [30]. The plot is made by interpolating the value of each measured depth of maximum between theoretical curves for protons and iron on a plot of $X_{\max }$ vs energy (in this case using SIBYLL 2.1 [32]). The increasing fraction of heavy nuclei up to $10^{17} \mathrm{eV}$, for example, can be interpreted naturally as a rigiditydependent effect as Galactic accelerators reach their maximum energy. This is followed by an increase in the proton fraction that reaches a maximum (minimum in $\langle\ln (A)\rangle)$ at the ankle. The preliminary IceCube result $[19,31]$ is shown in red in Fig. 5. It agrees with the trend of the data up to $10^{17} \mathrm{eV}$, but remains at a high level above that energy, although with large uncertainties.

In his presentation Ptuskin [33] reviewed the standard model of cosmic-ray origin, with Galactic supernovae of different types producing the cosmic-ray spectrum observed at Earth up to $\sim 10^{17} \mathrm{eV}$ and an extra-galactic component dominating above $10^{18} \mathrm{eV}$. Analysis of lowenergy iron isotopes [34] shows that there must have been at least two nearby supernova remnants within one or two million years of each other. The general picture of supernovae in the disk of the Galaxy exploding randomly and each accelerating cosmic rays for a period of time short

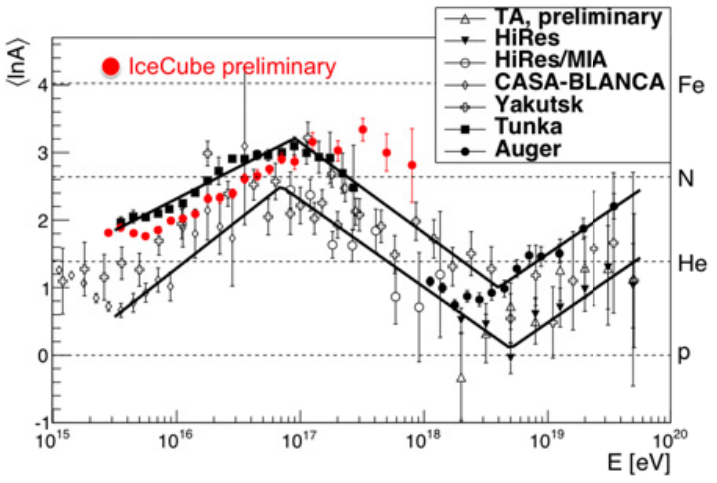

Figure 5. Mean value of the natural logarithm of the primary mass as a function of primary air shower energy. The plot is from the paper of Kampert \& Unger [30]), and the solid lines indicate the ranged of data summarized there. Preliminary composition data from IceCube [31] are interpolated in the same way and added to the Kampert \& Unger plot in red.

compared to the propagation time is explored in Ref. [35]. The contribution of a particular supernova to the cosmicray flux currently observed at Earth will be determined by the energy-dependent residence time of cosmic-rays in the Galaxy coupled with time of the explosion and its distance from Earth. Such considerations also have implications for the observed anistropy of cosmic rays [36], which will reflect to some extent the particular history of supernova explosions in the Milky Way.

\section{Multi-messenger astronomy}

Photons and neutrinos, being electrically neutral, propagate from their sources in straight lines, unlike charged cosmic rays, which are bent in Galactic and cosmic magnetic fields. Therefore, to the extent that cosmicrays interact in or near their sources, gamma-rays and neutrinos should be good probes of cosmic-ray sources. Neutrinos have the extra advantage of not being affected by interactions in transit (and the corresponding disadvantage of being difficult to detect). High-energy gamma-rays can be produced electromagnetically, for example by inverse Compton scattering by electrons, as well as from decay of $\pi^{0}$ produced in hadronic interactions. Neutrinos can only be produced by hadronic interactions. Just as atmospheric neutrinos are produced by interactions of cosmic rays in the atmosphere, any high-energy neutrino 

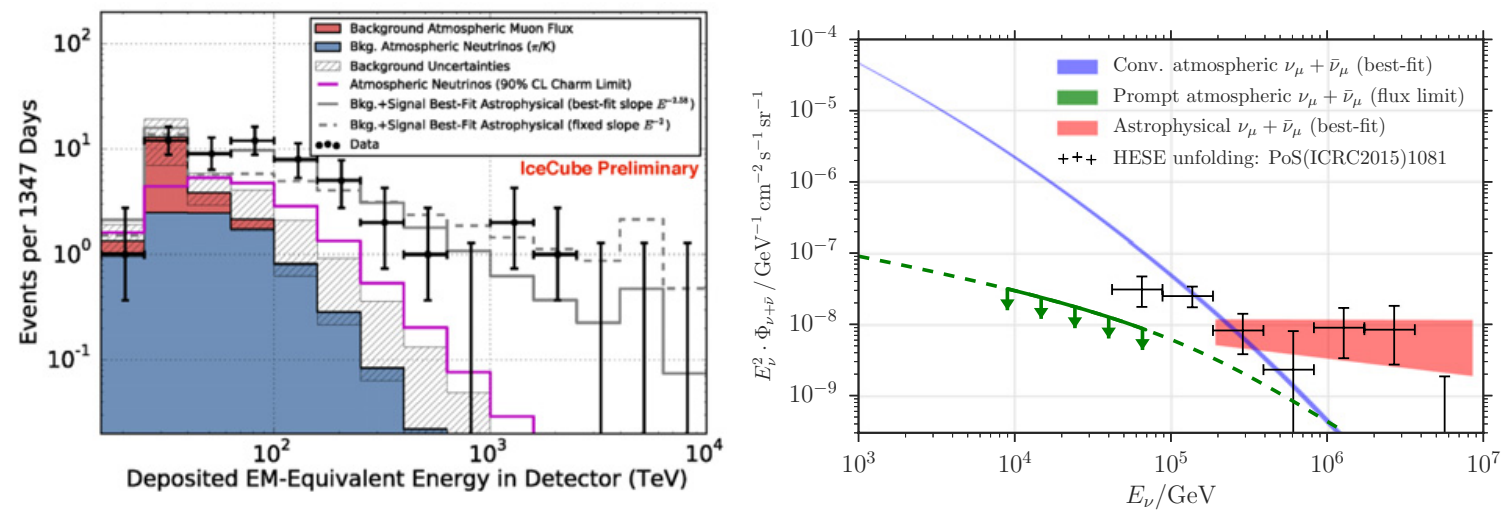

Figure 6. Spectrum of high energy neutrinos from IceCube, Left: 4-year HESE analysis [37]; Right: 6-year upward muon analysis [42].

of extraterrestrial origin will have been produced by the interaction of a proton (or less likely a nucleus) somewhere in the cosmos. The interaction may be with gas or by photo-pion production, but in both cases gamma-rays would be produced through the corresponding neutral pion channel. (The possibility of $\gamma$-rays and neutrinos from dark matter is not considered here.)

\subsection{High-energy neutrinos in IceCube}

Figure 6 shows the observation by IceCube of a hard spectrum of astrophysical neutrinos extending above the steeply falling background of atmospheric neutrinos. The signal is seen consistently in two channels. The highenergy starting event (HESE) analysis [38] requires the event to start well inside the instrumented volume of IceCube in such a way that the outer layers of optical modules exclude atmospheric muons from above, as well as atmospheric neutrinos of sufficiently high energy to be accompanied by a muon $[39,40]$. The astrophysical signal can be identified to lower energy by increasing the veto region and correspondingly reducing the size of the fiducial volume [41]. In the upward muon analysis [42], the Earth is used as a shield to exclude atmospheric muons. The signal then consists mainly of neutrino-induced muons. The astrophysical component is distinguished only by its hard spectrum extending to high energy.

Accompanying each of the neutrino analyses is a list of the highest energy events with their locations in the sky (right ascension and declination). In the case of the HESE analysis, the event type is given as cascade ( $v_{e}$ and most $\left.v_{\tau}\right)$ or track $\left(v_{\mu}\right)$. The four-year sample includes 54 events with deposited energy of $30 \mathrm{TeV}$ and higher, of which fewer than twenty are background. For the upward muon analysis, all events are tracks, and 29 events with estimated muon energy $E_{v}>200 \mathrm{TeV}$ are singled out as being the most likely to be of astrophysical origin. Sky maps are made for both cases, but no source of high significance emerges. The most energetic event in the upward muon sample has a deposited energy in the detector of $2.6 \pm 0.3 \mathrm{PeV}$ and an estimated muon energy of $4.5 \mathrm{PeV}$. The probability distribution for the parent neutrino energy peaks just below $10 \mathrm{PeV}$ [42].

The spectral index that characterizes the astrophysical flux depends on the energy range of the analysis, and the possibility of a break and or a cutoff in the spectrum is not ruled out. A combined maximum-likelihood analysis of the high-energy astrophysical neutrino flux measured with several IceCube analyses gives a value of the energy flux of all neutrino flavors of

$$
E_{v}^{2} \frac{\mathrm{d} N_{v}}{\mathrm{~d} E_{v}} \approx 2 \times 10^{-8} \mathrm{GeV} \mathrm{cm}^{-2} \mathrm{sr}^{-1} \mathrm{~s}^{-1}
$$

at $E_{v}=1 \mathrm{PeV}$ assuming the spectrum is a single power law [43]. The fitted differential spectral index, is $-2.5 \pm 0.09$. It is interesting to note from Fig. 1 that the energy flux of cosmic rays has the same value at the ankle, which may be interpreted as indicating that the energy flux of the extragalactic population of cosmic rays is somehow related to the astrophysical neutrino flux, as suggested by Waxman and Bahcall $[44,45]$.

In this context, it is interesting to note that detection of cosmogenic neutrinos from photo-pion production by interactions of ultra-high-energy cosmic rays with the CMB could resolve the origin of the cutoff of the cosmicray spectrum at $100 \mathrm{EeV}$. A search by IceCube for events of extremely high energy (EHE) with seven years of data [46] finds no neutrinos with energy $>10^{7} \mathrm{GeV}$. This result places significant constraints on some models of cosmogenic neutrinos. Because neutrinos produced by interactions of cosmic-rays with energy-per-nucleon $>10^{9} \mathrm{GeV}$ would produce neutrinos with $E_{v}>10^{7} \mathrm{GeV}$, the result also disfavors some models of astrophysical neutrinos associated with sources of ultra-high-energy cosmic rays.

\subsection{Extragalactic source candidates}

The tables of high-energy neutrinos $[38,42]$ constitute a small fraction of the $\sim 100,000$ neutrinos per year reconstructed in IceCube. Most of these are atmospheric neutrinos. Nevertheless, the most sensitive searches for point sources of astrophysical neutrinos use the larger data samples that extend down to the $\mathrm{TeV}$ energy range. Maximum likelihood techniques that take account of the angular resolution and the deposited energy of each event are used to look for point sources of neutrinos emerging from the smooth foreground of atmospheric neutrinos. An all-sky analysis produces a sky map of significances. In addition, a list of sources selected, largely on the basis of gamma-ray observations, to be likely neutrino sources is investigated. The most sensitive search so far [47] uses seven years of data and finds no source of high significance in the all-sky analysis and places upper limits on the targeted sources. 
The relation between a total observed intensity from all directions and the number of events to be expected from nearby sources is addressed in general in [48] and in the context of the IceCube discovery in [49]. Upper limits on extragalactic sources, together with an assumption about the cosmological evolution of the source class, lead to a lower limit on the density of sources. A recent analysis [50] of the total astrophysical intensity of neutrinos and the current upper limits of IceCube suggests that the density of extra-galactic sources should be $>10^{-7}(\mathrm{Mpc})^{-3}$. Blazars and Gamma-Ray Bursts (GRB) are among the rare, luminous sources that are being constrained by this line of argument [51]. Moreover, there are experimental upper limits from IceCube on the contribution to the IceCube signal of both blazars [52] and GRBs [53].

Starburst galaxies are relatively abundant and have been proposed as a likely source of neutrinos [54]. The idea is that, because of the greater rate of supernova explosions and the turbulence they generate, there would be a higher intensity of cosmic rays produced and they would be confined in the host galaxy sufficiently long that most protons would interact (calorimetric limit). Since the target for the neutrino production is gas in the galaxy, the spectrum of neutrinos and gamma-rays would extend down to low energy. Fermi observations of the diffuse gamma background [55] have been used to constrain the starburst model $[56,57]$. In order to avoid producing more diffuse gamma-radiation than observed, the spectra of cosmic-ray induced neutrinos and photons must have a hard spectrum (differential spectral index $-\alpha$ with $\alpha<2.2$ [56]. On the other hand, since most of the isotropic gamma-radiation comes from blazars [58], it would be surprising if there were not also neutrinos at some level [59].

Another possible neutrino source, discussed at this conference by Ptuskin [33], is Type IIn supernovae in external galaxies [60]. These are rare core-collapse supernovae that accelerate protons into the dense wind of the progenitor star. The environment of the progenitor wind is sufficiently dense that the SN expansion slows down on a time scale of 30 years. Like the starburst scenario, the target for neutrino (and gamma-ray) production is gas, so the spectra extend to low energy and the Fermi isotropic gamma limit applies (so the accelerated spectrum of protons must be hard). A maximum energy of $10^{17} \mathrm{eV} /$ proton is found, which is sufficient to produce neutrinos up to $\sim \mathrm{PeV}$ (but not beyond). Assuming a rate of $1 \%$ of all core collapse supernovae, a diffuse spectrum in rough agreement with the IceCube spectrum is found. Moreover, two tracklike events are in the direction of known Type IIn supernovae. HESE event 47 with $74 \mathrm{TeV}$ deposited energy [37] is $1.35^{\circ}$ from SNIIn 2005bx and event 11 of the upward muon sample with an estimated energy of $240 \mathrm{TeV}$ [42] is $0.3^{\circ}$ from SNIIn 2005jq.

\subsection{Neutrinos and $\gamma$-rays from the Milky Way}

Several of the events in the high-energy samples come from near the Galactic plane and could be of local origin in the Milky Way [61]. In fact a local component is expected because both neutrinos and photons are produced by cosmic-ray interactions of cosmic rays with interstellar gas during their diffusive propagation in the Galaxy. In his paper on neutrinos, Stecker [62] pointed out that these neutrinos would have the same spectrum as that of the primary cosmic rays $(\alpha \approx 2.7)$. At high energy the spectrum of atmospheric neutrinos is one power steeper, so the Galactic neutrinos should become relatively more prominent as energy increases. In addition, the cosmic-ray spectrum in the central region of the Galaxy could have a harder spectrum than observed locally at Earth [63].

The Galactic diffuse $\gamma$ emission is measured up to $\approx 100 \mathrm{GeV}$ by Fermi [64]. It is concentrated along the Galactic ridge and is mostly from $\pi^{0}$-decay. The ARGO-YBJ measurements of the diffuse $\gamma$-emission up to a $\mathrm{TeV}$ are consistent with an extension of the Fermi measurement [65]. The corresponding spectrum of $v_{\mu}+\bar{v}_{\mu}$ should have the same shape and a magnitude of $2 / 3$ that of the gamma-rays at production and $1 / 3$ after oscillations [7]. In the presentation at this conference [8] the high intensity of gamma-rays from the region of the Cygnus Cocoon [66] was also described. This too should have a counterpart in neutrinos. The ANTARES neutrino detector has placed a limit [67] on the intensity of multi$\mathrm{TeV}$ neutrinos from the Galactic plane that is approaching the higher prediction of Ref. [63].

Using gamma-ray data from Fermi-LAT as a template, predictions for Galactic neutrinos in the $\mathrm{TeV}$ to $\mathrm{PeV}$ range are examined in detail in Ref. [68]. The conclusion is that less than $10 \%$ of the high-energy events are of Galactic origin. Limits are also placed on the Galactic contributions in the $\mathrm{TeV}$ range.

\section{Summary and outlook}

Understanding the origin of ultra-high energy cosmic rays and whether their sources also produce neutrinos remains a key question [46]. It is possible that the sources of the astrophysical neutrinos seen by IceCube are not the sources of UHECR. The current status of neutrinos and cosmic-rays observed by IceCube is the subject of a recent review [69].

To improve the ability to identify the sources of highenergy astrophysical neutrinos, IceCube has started a realtime alert system [70]. Its goal is to maximize the ability to find electromagnetic counterparts of astrophysical neutrinos seen in IceCube. There are several types of alerts. Single track-like events (with good pointing and high energy) in the HESE and EHE samples generate alerts. Alerts for follow-up by optical, X-ray and $\gamma$-ray detectors trigger on multiple events from a single point in the sky in a limited time interval.

The discovery of high-energy astrophysical neutrinos motivates efforts to accumulate more data with new and larger detectors. Construction of KM3NeT is starting, with the deployment of test lines at the Italian site, Capo Passero [71] and a Letter of Intent [72]. The proposal is to build a high-energy unit called ARCA at Capo Passero and a densely instrumented array called ORCA at the French site near Marseille [71]. The first cluster of an expansion of Baikal to a Gigaton Volume Detector (Baikal-GVD) [73] was installed in 2015. Plans for a next generation IceCubeGen2 are underway $[74,75]$. IceCube-Gen2 will include a densely instrumented subarray for neutrino physics [76].

The high-energy expansion of IceCube-Gen2 aims to increase the target volume by an order of magnitude. To reach the much larger volumes needed to detect a large number of cosmogenic neutrinos, the radio technique is being pursued. The first detectors of ARIANNA [77] 
and of ARA [78] prototypes are already in operation in Antarctica. A review of the current status of high-energy neutrino astronomy that covers current and future detectors and the science they will address is in preparation [79].

I thank John Matthews for permission to use his composite spectrum of Telescope Array and TALE (Fig. 4), and I thank Javier Gonzalez for adding the IceCube data points in Fig. 5. I thank Christopher Wiebusch for helpful comments on a draft version of this paper, and I acknowledge support from the U.S. National Science Foundation (PHY-1505990).

\section{References}

[1] T. Pierog, These Proceedings

[2] O. Adriani et al. [PAMELA Collaboration], "PAMELA Measurements of Cosmic-ray Proton and Helium Spectra," Science 332, 69 (2011) [arXiv:1103.4055 [astro-ph.HE]]

[3] M. Aguilar et al. [AMS Collaboration], "Precision Measurement of the Proton Flux in Primary Cosmic Rays from Rigidity $1 \mathrm{GV}$ to $1.8 \mathrm{TV}$ with the Alpha Magnetic Spectrometer on the International Space Station," Phys. Rev. Lett. 114, 171103 (2015)

[4] M. Aguilar et al. [AMS Collaboration], "Precision Measurement of the Helium Flux in Primary Cosmic Rays of Rigidities $1.9 \mathrm{GV}$ to $3 \mathrm{TV}$ with the Alpha Magnetic Spectrometer on the International Space Station,” Phys. Rev. Lett. 115(21), 211101 (2015)

[5] A. D. Panov et al., "Energy Spectra of Abundant Nuclei of Primary Cosmic Rays from the Data of ATIC-2 Experiment: Final Results,' Bull. Russ. Acad. Sci. Phys. 73, 564 (2009) [arXiv: 1101.3246 [astro-ph.HE]]

[6] H. S. Ahn et al., "Energy spectra of cosmic-ray nuclei at high energies," Astrophys. J. 707, 593 (2009) [arXiv:0911.1889 [astro-ph.HE]]

[7] Thomas K. Gaisser, Ralph Engel and Elisa Resconi, Cosmic Rays and Particle Physics (Cambridge University Press, 2016)

[8] G. Di Sciascio, this conference

[9] L. Kuzmichev, this conference

[10] B. Bartoli et al. [ARGO-YBJ and LHAASO Collaborations], "Knee of the cosmic hydrogen and helium spectrum below $1 \mathrm{PeV}$ measured by ARGOYBJ and a Cherenkov telescope of LHAASO," Phys. Rev. D 92(9), 092005 (2015) [arXiv: 1502.03164 [astro-ph.HE]]

[11] T. Antoni et al. [KASCADE Collaboration], "KASCADE measurements of energy spectra for elemental groups of cosmic rays: Results and open problems," Astropart. Phys. 24, 1 (2005) [astro-ph/0505413]

[12] A. Haungs, this conference

[13] J. R. Hoerandel, "On the knee in the energy spectrum of cosmic rays," Astropart. Phys. 19, 193 (2003) [astro-ph/0210453]

[14] T. K. Gaisser, "Spectrum of cosmic-ray nucleons, kaon production, and the atmospheric muon charge ratio," Astropart. Phys. 35, 801 (2012) [arXiv:1111.6675 [astro-ph.HE]]

[15] M. G. Aartsen et al. [IceCube Collaboration], "The IceCube Neutrino Observatory -
Contributions to ICRC 2015 Part III: Cosmic Rays," arXiv:1510.05225 [astro-ph.HE], pp. 37-44

[16] W. D. Apel et al., "The spectrum of high-energy cosmic rays measured with KASCADE-Grande," Astropart. Phys. 36, 183 (2012)

[17] V. V. Prosin, this conference

[18] V. V. Prosin et al., "Tunka-133: Results of 3 year operation,” Nucl. Instrum. Meth. A 756, 94 (2014)

[19] H. Dembinski, this conference

[20] M. G. Aartsen et al. [IceCube Collaboration], "Measurement of the cosmic ray energy spectrum with IceTop-73," Phys. Rev. D 88(4), 042004 (2013) [arXiv:1307.3795 [astro-ph.HE]]

[21] W. D. Apel et al., "Ankle-like Feature in the Energy Spectrum of Light Elements of Cosmic Rays Observed with KASCADE-Grande," Phys. Rev. D 87, 081101 (2013) [arXiv:1304.7114 [astro-ph.HE] ]

[22] J. Matthews, this conference

[23] D. Martello, this conference

[24] K. Greisen, "End to the cosmic ray spectrum?," Phys. Rev. Lett. 16, 748 (1966)

[25] G. T. Zatsepin and V. A. Kuzmin, "Upper limit of the spectrum of cosmic rays,” JETP Lett. 4, 78 (1966) [Pisma Zh. Eksp. Teor. Fiz. 4, 114 (1966)]

[26] A. M. Hillas, "The Origin of Ultrahigh-Energy Cosmic Rays," Ann. Rev. Astron. Astrophys. 22, 425 (1984)

[27] A. di Matteo et al. in "The Pierre Auger observatory: Contributions to the 34th international Cosmic aAy Conference (ICRC 2015)," (arXiv:1509.03732) p. $103-110$

[28] R. U. Abbasi et al. [Telescope Array Collaboration], "Indications of Intermediate-Scale Anisotropy of Cosmic Rays with Energy Greater Than $57 \mathrm{EeV}$ in the Northern Sky Measured with the Surface Detector of the Telescope Array Experiment," Astrophys. J. 790, L21 (2014) [arXiv: 1404.5890 [astro-ph.HE]]

[29] A. Aab et al. [Pierre Auger Collaboration], "The Pierre Auger Observatory Upgrade - Preliminary Design Report," arXiv: 1604.03637 [astro-ph.IM]

[30] K. H. Kampert and M. Unger, "Measurements of the Cosmic Ray Composition with Air Shower Experiments," Astropart. Phys. 35, 660 (2012) [arXiv: 1201.0018 [astro-ph.HE]]

[31] K. Rawlins [IceCube Collaboration], J. Phys. Conf. Ser. 718(5), 052033 (2016). doi: 10.1088/17426596/718/5/052033

[32] E. J. Ahn, R. Engel, T. K. Gaisser, P. Lipari, T. Stanev, "Cosmic ray interaction event generator SIBYLL 2.1," Phys. Rev. D 80, 094003 (2009) [arXiv:0906.4113 [hep-ph]]

[33] V. Ptuskin, this conference

[34] T. Hams et al., "Elemental Abundances of UltraHeavy GCRs measured by SuperTIGER and ACECRIS and the Origin of Galactic Cosmic Rays," PoS ICRC 2015, 038 (2016)

[35] P. Blasi and E. Amato, "Diffusive propagation of cosmic rays from supernova remnants in the Galaxy. I: spectrum and chemical composition," JCAP 1201, 010 (2012) [arXiv: 1105.4521 [astro-ph.HE] ] 
[36] P. Blasi and E. Amato, "Diffusive propagation of cosmic rays from supernova remnants in the Galaxy. II: anisotropy," JCAP 1201, 011 (2012) [arXiv: 1105.4529 [astro-ph.HE]]

[37] M. G. Aartsen et al. [IceCube Collaboration], "The IceCube Neutrino Observatory - Contributions to ICRC 2015 Part II: Atmospheric and Astrophysical Diffuse Neutrino Searches of All Flavors," arXiv:1510.05223 [astro-ph.HE]

[38] M. G. Aartsen et al. [IceCube Collaboration], "Observation of High-Energy Astrophysical Neutrinos in Three Years of IceCube Data," Phys. Rev. Lett. 113, 101101 (2014) [arXiv:1405.5303 [astro-ph.HE]]

[39] S. Schonert, T. K. Gaisser, E. Resconi and O. Schulz, "Vetoing atmospheric neutrinos in a high energy neutrino telescope," Phys. Rev. D 79, 043009 (2009) [arXiv:0812.4308 [astro-ph]]

[40] T. K. Gaisser, K. Jero, A. Karle, J. van Santen, "Generalized self-veto probability for atmospheric neutrinos," Phys. Rev. D 90(2), 023009 (2014) [arXiv:1405.0525 [astro-ph.HE]]

[41] M. G. Aartsen et al. [IceCube Collaboration], "Atmospheric and astrophysical neutrinos above $1 \mathrm{TeV}$ interacting in IceCube," Phys. Rev. D 91(2), 022001 (2015) [arXiv: 1410.1749 [astro-ph.HE]]

[42] M. G. Aartsen et al. [IceCube Collaboration], "Observation and Characterization of a Cosmic Muon Neutrino Flux from the Northern Hemisphere using six years of IceCube data," Astrophys. J. 833(1), 3 (2016) [arXiv:1607.08006 [astro-ph.HE]]

[43] M. G. Aartsen et al. [IceCube Collaboration], "A combined maximum-likelihood analysis of the high-energy astrophysical neutrino flux measured with IceCube," Astrophys. J. 809(1), 98 (2015) [arXiv:1507.03991 [astro-ph.HE]]

[44] E. Waxman, J. N. Bahcall, "High-energy neutrinos from astrophysical sources: An Upper bound," Phys. Rev. D 59, 023002 (1999) [hep-ph/9807282]

[45] J. N. Bahcall, E. Waxman, "High-energy astrophysical neutrinos: The Upper bound is robust," Phys. Rev. D 64, 023002 (2001) [hep-ph/9902383]

[46] M. G. Aartsen et al. [IceCube Collaboration], "Constraints on Ultrahigh-Energy Cosmic-Ray Sources from a Search for Neutrinos above $10 \mathrm{PeV}$ with IceCube," Phys. Rev. Lett. 117(24), 241101 (2016) [arXiv:1607.05886 [astro-ph.HE]]

[47] M. G. Aartsen et al. [IceCube Collaboration], "All-sky search for time-integrated neutrino emission from astrophysical sources with 7 years of IceCube data," Astrophys. J. 835(2), 151 (2017) [arXiv:1609.04981 [astro-ph.HE]]

[48] P. Lipari, "Proton and Neutrino Extragalactic Astronomy," Phys. Rev. D 78, 083011 (2008) [arXiv:0808.0344 [astro-ph]]

[49] M. Ahlers and F. Halzen, "Pinpointing Extragalactic Neutrino Sources in Light of Recent IceCube Observations," Phys. Rev. D 90(4), 043005 (2014) [arXiv:1406.2160 [astro-ph.HE]]

[50] K. Murase, E. Waxman, "Constraining HighEnergy Cosmic Neutrino Sources: Implications and Prospects," Phys. Rev. D 94(10), 103006 (2016) [arXiv:1607.01601 [astro-ph.HE]]
[51] M. Kowalski, "Status of High-Energy Neutrino Astronomy," J. Phys. Conf. Ser. 632(1), 012039 (2015) [arXiv:1411.4385 [astro-ph.HE]]

[52] M. G. Aartsen et al. [IceCube Collaboration], "The contribution of Fermi-2LAC blazars to the diffuse TeV-PeV neutrino flux," Astrophys. J. 835(1), 45 (2017) [arXiv: 1611.03874 [astro-ph.HE]]

[53] M. G. Aartsen et al. [IceCube Collaboration], "An All-Sky Search for Three Flavors of Neutrinos from Gamma-Ray Bursts with the IceCube Neutrino Observatory," Astrophys. J. 824(2), 115 (2016) [arXiv:1601.06484 [astro-ph.HE]]

[54] A. Loeb, E. Waxman, "The Cumulative background of high energy neutrinos from starburst galaxies," JCAP 0605, 003 (2006) [astro-ph/0601695]

[55] M. Ackermann et al. [Fermi-LAT Collaboration], "The spectrum of isotropic diffuse gamma-ray emission between $100 \mathrm{MeV}$ and $820 \mathrm{GeV}$," Astrophys. J. 799, 86 (2015) [arXiv:1410.3696 [astro-ph.HE]]

[56] K. Murase, M. Ahlers and B. C. Lacki, "Testing the Hadronuclear Origin of PeV Neutrinos Observed with IceCube,” Phys. Rev. D 88(12), 121301 (2013) [arXiv:1306.3417 [astro-ph.HE]]

[57] K. Bechtol, M. Ahlers, M. Di Mauro, M. Ajello, J. Vandenbroucke, "Evidence against star-forming galaxies as the dominant source of IceCube neutrinos,"arXiv:1511.00688 [astro-ph.HE]

[58] M. Ackermann et al. [Fermi-LAT Collaboration], "Resolving the Extragalactic $\gamma$-Ray Background above $50 \mathrm{GeV}$ with the Fermi Large Area Telescope,” Phys. Rev. Lett. 116(15), 151105 (2016) [arXiv:1511.00693 [astro-ph.Co]]

[59] E. Resconi, S. Coenders, P. Padovani, P. Giommi, L. Caccianiga, "Connecting blazars with ultra high energy cosmic rays and astrophysical neutrinos," arXiv:1611.06022 [astro-ph.HE]

[60] V. N. Zirakashvili and V. S. Ptuskin, "Type IIn supernovae as sources of high energy astrophysical neutrinos," Astropart. Phys. 78, 28 (2016) [arXiv: 1510.08387 [astro-ph.HE]]

[61] A. Neronov and D. V. Semikoz, "Evidence the Galactic contribution to the IceCube astrophysical neutrino flux," Astropart. Phys. 75, 60 (2016) [arXiv:1509.03522 [astro-ph.HE]]

[62] F. Stecker, "Diffuse fluxes of cosmic high-energy neutrinos," Astrophys. J. 228, 919 (1979)

[63] D. Gaggero, D. Grasso, A. Marinelli, A. Urbano and M. Valli, "The gamma-ray and neutrino sky: A consistent picture of Fermi-LAT, Milagro, and IceCube results," Astrophys. J. 815, no. 2, L25 (2015) [arXiv: 1504.00227 [astro-ph.HE]]

[64] M. Ackermann et al. [Fermi-LAT Collaboration], "Fermi-LAT Observations of the Diffuse GammaRay Emission: Implications for Cosmic Rays and the Interstellar Medium," Astrophys. J. 750, 3 (2012) [arXiv:1202.4039 [astro-ph.HE]]

[65] B. Bartoli et al. [ARGO-YBJ Collaboration], "Study of the Diffuse Gamma-ray Emission From the Galactic Plane With ARGO-YBJ," Astrophys. J. 806, 20 [arXiv: 1507.06758 [astro-ph.IM] ]

[66] M. Ackermann et al., "A Cocoon of Freshly Accelerated Cosmic Rays Detected by Fermi in 
the Cygnus Superbubble," Science 334(6059), 1103 (2011)

[67] S. Adrian-Martinez et al. [ANTARES Collaboration], "Constraints on the neutrino emission from the Galactic Ridge with the ANTARES telescope," Phys. Lett. B 760, 143 (2016) [arXiv: 1602.03036 [astro-ph.HE]]

[68] M. Ahlers, Y. Bai, V. Barger and R. Lu, "Galactic neutrinos in the $\mathrm{TeV}$ to $\mathrm{PeV}$ range," Phys. Rev. D 93(1), 013009 (2016) [arXiv:1505.03156 [hep-ph] ]

[69] M. G. Aartsen et al. [IceCube Collaboration], "Neutrinos and Cosmic Rays Observed by IceCube," arXiv: 1701.03731 [astro-ph.HE]

[70] M. G. Aartsen et al. [IceCube Collaboration], "The IceCube Realtime Alert System," arXiv:1612.06028 [astro-ph.HE]

[71] J. Brunner, for the KM3NeT Collaboration, presentation at RICAP 2016

[72] S. Adrian-Martinez et al. [KM3Net Collaboration], "Letter of intent for KM3NeT 2.0," J. Phys. G 43(8), 084001 (2016) [arXiv:1601.07459 [astro-ph. IM] ]

[73] A. D. Avrorin et al. [BAIKAL Collaboration], "The prototyping/early construction phase of the BAIKAL-GVD project," Nucl. Instrum.
Meth. A 742, 82 (2014) [arXiv:1308.1833 [astro-ph. IM] ]

[74] M. G. Aartsen et al. [IceCube Collaboration], "IceCube-Gen2: A Vision for the Future of Neutrino Astronomy in Antarctica," arXiv:1412.5106 [astro-ph.HE]

[75] M. G. Aartsen et al. [IceCube Collaboration], "IceCube-Gen2 - The Next Generation Neutrino Observatory at the South Pole: Contributions to ICRC 2015," arXiv: 1510.05228 [astro-ph. IM]

[76] M. G. Aartsen et al. [IceCube Collaboration], "PINGU: A Vision for Neutrino and Particle Physics at the South Pole," arXiv:1607.02671 [hep-ex]

[77] S. W. Barwick et al., "Radio detection of air showers with the ARIANNA experiment on the Ross Ice Shelf," arXiv:1612.04473 [astro-ph. IM]

[78] P. Allison et al. [ARA Collaboration], "Performance of two Askaryan Radio Array stations and first results in the search for ultrahigh energy neutrinos," Phys. Rev. D 93(8), 082003 (2016) [arXiv: 1507.08991 [astro-ph.HE] ]

[79] Neutrino Astronomy: current status, future prospects (ed. Thomas Gaisser \& Albrecht Karle, World Scientific 2017) 